fournal of Medical Genetics (1973). 10, 122.

\title{
Photographic Techniques for Recording Chromosome Banding Patterns
}

\author{
N. R. DAVIDSON \\ Medical Research Council, Clinical and Population Cytogenetics Unit, Western General Hospital, Edinburgh
}

The advent of the new staining techniques for chromosome banding patterns has introduced additional problems to the photomicrographer. Before the variations in photomicrography can be considered, it is of paramount importance that the quality of the chromosome preparations is of a sufficiently good standard. It is pointless to follow through strict microscope and photographic procedure if in the first place cell quality is poor. With so many types of emulsions available to the photomicrographer it is necessary to have a knowledge of their characteristics and utilize them to the most appropriate means. In order to produce quality photomicrographs this knowledge is used in conjunction with perfect microscope and photographic technique.

\section{Microscope Technique}

Although this article is primarily concerned with photographic techniques, some points about microscope technique should be considered since the production of high quality photomicrographs is partially dependent upon good basic microscopy. Manufacturers of research microscopes have reached a high standard in production both in optics and in design. Automatic photomicroscopes are available which have built-in metering systems, such as a photomultiplier or selenium cell, and are often coupled with automatic 'winding-on' facilities, but it must be stressed that they should not be used as simple 'press-button' instruments with the added prayer that the apparatus will do the rest. Photomicroscopes of this nature are an asset, especially when photographing Q-banded preparations of quinacrine-stained chromosomes, but it must be

Received 29 January 1973. borne in mind that they only simplify exposure estimation and film 'winding-on', consequently the correct setting up of the microscope and calibration still applies. In the photomicrography of chromosomes, the objectives generally used are oil immersion $\times 90$ to $\times 100$ which together with $\times 8$ or $\times 12$ eyepieces and a microscope tube factor of 1.25 will produce a final magnification of around 1200 diameters. This is near the limit of useful magnification in the light microscope and therefore any slight misadjustment in setting up will deteriorate the image quality. The resolution of objectives is governed by the numerical aperture (NA) engraved on the lens mount, eg, the NA of a $\times 100$ objective is approximately $1 \cdot 30$. Although the resolution is governed by the NA of the objective, maximum resolution is only possible if a condenser is employed with an equivalent numerical aperture NA 1.25. Thus it is evident that the top lens of the sub-stage condenser determines the limit for maximum resolution and in order to produce optimum results, ideally the numerical aperture of the condenser should be matched with the numerical aperture of the objective. This can be achieved by using an oil cap condenser NA 1.25 and the use of immersion oil on the top lens.

In the majority of modern microscopes the lamp source is built in and the conditions of Köhler's illumination are therefore applied, but if the lamp source is separate careful attention must be made in applying Köhler's illumination. The lamp should be correctly aligned to produce even illumination before any photomicrography can begin.

Once an appropriate cell has been selected the following steps should be carried out before each exposure.

1. The cell is focused with $\times 100$ objective. 
2. The sub-stage condenser is racked down and a drop of immersion oil applied to top lens.

3. The field diaphragm is stopped down.

4. The sub-stage is racked up slowly until a sharp image of the field diaphragm is produced in the plane of the focused cell.

5. The field diaphragm is centred and opened just beyond image field.

6. The sub-stage iris diaphragm is stopped down by about one third.

7. The focus is rechecked.

These steps apply to all bright field work and must be employed for each exposure if maximum resolution is to be obtained.

\section{Camera Apparatus and Calibration}

The range of camera equipment available to the photomicrographer is vast, whether it be a simple $35 \mathrm{~mm}$ camera, a fully automatic $35 \mathrm{~mm}$ camera, or roll and sheet film camera; basically the same techniques apply. It is advisable that a test strip should be taken either when new equipment is used or when a new staining technique is introduced. This test strip is made by giving the film a series of exposures which range from under-exposure to overexposure with the exposures being made under constant conditions.

In order to produce a valid test strip, from which the ideal negative can be selected, all conditions throughout must be stable. The light source should be set at a constant voltage and any filters necessary are placed in the filter holder. Varying exposures are now given to the test film by varying the exposure time on manual cameras or by changing the ASA settings on fully automatic cameras. All data concerning film, filters, lamp voltage, exposures, and development must be recorded for future use. It is still necessary to calibrate fully automatic cameras as conditions vary from apparatus to apparatus. Test films should be repeated if any condition is altered at a later date, eg, change of film developer, staining technique, etc. After washing and drying the test strip the ideal negative is selected either by printing the negatives onto a normal grade of paper and judging the correct print, or by experience. The time spent on this initial calibration of the apparatus will save many frustrating moments later.

\section{Photographic Techniques}

One of the problems encountered when photographing chromosomes is the apparent lack of contrast in some preparations. Contrast can be increased by photography but care should be exer- cised to avoid excessive contrast which can result in prints of chromosomes that resemble those of ink drawings. The problem of staining variation arises with all biological material. In the case of banded chromosome preparations this problem is magnified to an even greater extent where the cytologist's aim is to produce very pale and very dense staining regions within the same chromosome. It is therefore important to note that when calibrating the apparatus a cell of average staining is chosen for the preparation of the test strip. Minor deviations from the normal staining reaction can be tolerated and adjusted at the printing stage by printing onto a harder grade of paper.

With the introduction of quinacrine fluorescence (Q-banding) and the various Giemsa (G- and Cbanding) techniques photomicrography has to be adapted for each of these staining reactions. In order to produce high quality photomicrographs a knowledge of the various types of emulsions and their particular characteristics is necessary. Generally emulsions can be subdivided into three groups: slow, medium, and fast. The sensitivity or speed of an emulsion is proportional to the grain size, therefore slow emulsions render the finest grain size and offer a high resolving power. Film speed is inversely proportional to contrast so as sensitivity increases contrast decreases. It follows that slow emulsions producing high contrast, a fine grain and a high resolving power, are to be preferred for photomicrography (Table I).

The contrast of the emulsion can also be affected by the type of developer employed. With so many types available it is advisable to adhere to the individual film manufacturer's recommendations. Time, temperature, and agitation must be consistent throughout all processing if uniform results are to be expected.

Photographic filters are an invaluable aid in photomicrography giving further control over the contrast of the specimen. Filters of a complementary colour to the staining reaction increase the contrast while those of the same colour will reduce

TABLE I

\begin{tabular}{l|rll}
\hline Emulsion (35 mm) & Speed Rating & & Staining Technique \\
\cline { 1 - 2 } Kodak Microfile 5669* & 6-12 ASA & Orcein and C-banding \\
Ilford Micro Neg Pan & 12-20 ASA & & Orcein and C-banding \\
Adox KB 14 & 20 ASA & G-banding and Q-banding \\
Kodak Panatomic-X & 32 ASA & & G-banding and Q-banding \\
Ilford Pan F & 50 ASA & G-banding and Q-banding \\
Adox KB 17 & 40 ASA & G-banding and Q-banding \\
\hline
\end{tabular}

* Available only in $100 \mathrm{ft}$ lengths. 


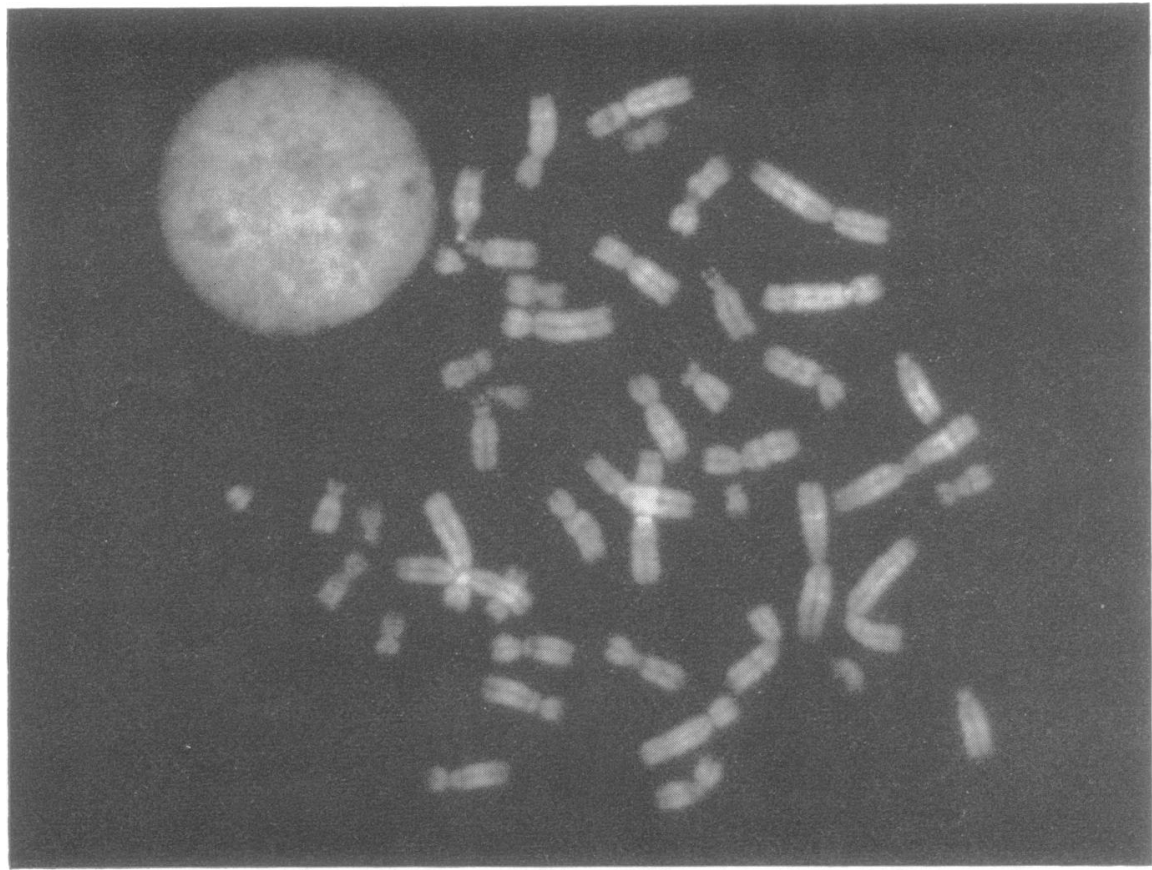

contrast. Stains normally used in the G- and Cbanding techniques vary from magenta to blue therefore the filters required are yellow, yellowgreen, and green. For example, a yellow-green filter $(560 \mathrm{~nm})$ is used for the average G-banded preparation except where the staining reaction is pale, then a green filter $(510 \mathrm{~nm})$ should be inserted to increase the contrast. Interference filters have an advantage over dyed-in-the-mass glass filters in that they produce a narrow transmission band but this gives rise to increased exposure times.

Q-Banding. Fluorescent photomicrography of banded patterns creates two main problems that govern the choice of emulsion. In fluorescent photomicrography exposure times are long, and since the staining reaction is unstable on exposure to blue light prolonged exposure can result in total loss of the initial banding pattern. It is therefore important to select an emulsion that is fast enough to allow the correct exposure to be made before any significant fading of the preparation results. Fast emulsions can be utilised giving extremely short exposure times, but negatives on these films exhibit graininess resulting, in a loss of image sharpness and because of their speed, the contrast is reduced.
Dependent upon the type of equipment, light source, exciter filters, etc the ideal emulsion to use would be Kodak Panatomic-X. If all trial exposures in an initial test strip are underexposed then a faster emulsion can be tried, eg, Ilford Pan F.

Development of these emulsions should be in a standard medium contrast developer such as Kodak D76, undiluted, for 8 minutes at $68^{\circ} \mathrm{F}$. Fluorescent negatives normally require to be printed on a grade above 'normal' grade bromide paper in order to enhance the banding pattern.

It is worth noting that immersion oil used in fluorescent photomicrography must be fluorescence free and, in order to reduce problems with glare and strong light, only the minimal quantities should be applied to give immersion with the slide.

The usual procedure in our laboratory for photomicrography of $\mathrm{Q}$-banded preparations is as follows:

Apparatus. Leitz Ortholux microscope and Orthomat camera.

Light Source. HBO 200-watt/4 mercury vapour lamp. Incident illumination is produced with a Ploem vertical illuminator at setting 3 .

Filters. Excitation filter BG12; Barrier filter $510 \mathrm{~nm}$.

Emulsion. Kodak Panatomic-X. 
Developer. Kodak D76 undiluted for 8 minutes at $68^{\circ} \mathrm{F}$. Agitation four times per minute.

Printing. Prints are made on Kodak grade 3 bromide paper and developed in Kodak D163 $(1+3)$ for 2 minutes.

G-Banding. G-banding produces varying densities along the arms of the chromosome and in order to record these densities, careful consideration must be given to the choice of emulsion. Inferior prints will result if a high contrast emulsion is employed due to the fact that the density range along the arms is compressed and therefore the clarity of the banding pattern is reduced or lost completely. Similar results would be produced if a negative of average contrast was printed onto a hard grade of paper. An emulsion of moderate contrast with a long tonal range capable of recording the banding densities is required. Kodak Panatomic-X is an ideal film for this purpose and should be developed in D76 undiluted for 8 minutes at $68^{\circ} \mathrm{F}$. (Kodak Data Book 5.) A yellow-green filter should be used for well stained preparations. If the staining reaction is pale and the final print quality poor with Panatomic-X, a slower film should be employed (see Table I).
A typical procedure for the photomicrography of G-banded preparations in our laboratory is as follows:

Apparatus. Leitz Ortholux microscope and Orthomat camera.

Light Source. 100-watt tungsten filament bulb. Normal brightfield illumination.

Filters. Interference filters $570 \mathrm{~nm}$ for well stained preparations.

Interference filter $560 \mathrm{~nm}$ for pale stained preparations.

Emulsion. Kodak Panatomic-X.

Developer. Kodak D76 undiluted for 8 minutes at $68^{\circ} \mathrm{F}$. Agitation four times per minute.

Printing. Prints are usually made on 'normal' (Kodak grade 2) bromide paper, depending on negative contrast, and developed in Kodak D163 $(1+3)$ for $2-2 \frac{1}{2}$ minutes.

C-Banding. Photomicrography of C-banded preparations requires a relatively high contrast emulsion. Satisfactory results can be obtained using Kodak Microfile Type 5669 and developing in D76 undiluted for 5 minutes at $68^{\circ} \mathrm{F}$. A yellow green filter should be used for well stained

FIG. 2. Photomicrograph of an ASG preparation showing G-banding. 
FIG. 3. Photomicrograph of a C-banded preparation.

preparations but for poorer stained preparations a green filter should be employed. If unsatisfactory results are obtained even with the use of a narrow cut green filter, the emulsion should be developed in Kodak DG10 developer $1+4$ for 5 minutes at $68^{\circ} \mathrm{F}$. This developer will produce a higher contrast than D76 and should be employed if the staining reaction is excessively poor.

The usual procedure in our laboratory for photomicrography of C-banded preparations is as follows:

Appparatus. Leitz Ortholux microscope and Orthomat camera.

Light Source. 100-watt tungsten filament bulb.

Filters. Interference filters $570-560 \mathrm{~nm}$ (approximately) or narrow cut green filter.

Emulsion. Kodak Recordak Microfile Type 5669.

Developer. D76 undiluted for 5 minutes at $68^{\circ} \mathrm{F}$. Agitation four times per minute.
Printing. Prints are normally made on Kodak grade 2 bromide paper and developed in Kodak D163 $(1+3)$ for 2 minutes.

With all photomicrography final print quality is mainly governed by the quality of the negative. It is therefore important that the photomicrographer should make a visual estimation of the contrast and density required in a negative in order to produce the optimum print.

I am very grateful to Miss Maureen O'Riordan for her helpful criticism.

\section{BIBLIOGRAPHY}

Christenson, L. P. (1965). Applied photography in chromosome studies. In Human Chromosome Methodology, ed. by J. J. Yunis, pp. 129-153. Academic Press, New York

Kodak Data Books 1-5. Kodak Ltd, London.

Runge, W. J. (1965). Bright field, phase contrast and fluorescence microscopy. In Human Chromosome Methodology, ed. by J. J. Yunis, pp. 111-128. Academic Press, New York.

Schenk, R. and Kistler, G. (1962). Photomicrography, translated by 